\title{
Comparison and unification of non-Hermitian and Lindblad approaches with applications to open quantum optical systems
}

\author{
Konstantin G. Zloshchastiev ${ }^{1}$ and Alessandro Sergi ${ }^{1,2}$ \\ 1 School of Chemistry and Physics, University of KwaZulu-Natal, Pietermaritzburg Campus, \\ Private Bag X01, Scottsville, Pietermaritzburg 3209, South Africa \\ ${ }^{2}$ National Institute for Theoretical Physics (NITheP), KwaZulu-Natal Node, South Africa
}

(Dated: Received: 2 April 2014 [JMO], 28 May 2014 [arXiv])

\begin{abstract}
We compare two approaches to open quantum systems, namely, the non-Hermitian dynamics and the Lindblad master equation. In order to deal with more general dissipative phenomena, we propose the unified master equation that combines the characteristics of both of these approaches. This allows us to assess the differences between them as well as to clarify which observed features come from the Lindblad or the non-Hermitian part, when it comes to experiment. Using a generic two-mode single-atom laser system as a practical example, we analytically solve the dynamics of the normalized density matrix operator. We study the two-level model in a number of cases (depending on parameters and types of dynamics), compute different observables and study their physical properties. It turns out that one is able not only to describe the different types of damping in dissipative quantum optical systems but also to mimic the undamped anharmonic oscillatory phenomena which happen in quantum systems with more than two levels (while staying within the framework of the analytically simple two-mode approximation).
\end{abstract}

PACS numbers: $42.50 . \mathrm{Nn}, 03.65 . \mathrm{Yz}, 03.65 . \mathrm{Aa}, 42.55 . \mathrm{Ah}$

\section{INTRODUCTION}

There are various theoretical methods that can be used in order to study open quantum systems. One can start by considering the totality of the degrees of freedom and afterwards focus, either analytically [1] or numerically [2], on a subset of relevant coordinates. However, the approach that is currently more popular, immediately integrates over the degrees of freedom of the environment and, for the Markovian systems, establishes the Lindblad master equation [3, 4]. Originally introduced in spin physics and quantum optics [5, [6], master equations have been applied to many dissipative quantum phenomena [7, 9].

There is yet another approach to open quantum systems that is based on a non-Hermitian extension of quantum mechanics (NHQM) $10-22$. In this approach the Hamiltonian is assumed to acquire an anti-Hermitian part which can be associated with dissipative effects. Both the Lindblad and non-Hermitian approaches are based on certain simplifying assumptions and have their own range of applicability. Moreover, one can find different physical motivations and theoretical advantages in their respective use: the Lindblad master equation is linear and permits the simple calculation of averages whereas non-Hermitian dynamics possesses a generalized canonical structure that leads to a well-defined classical limit [21].

In the present work, we compare the evolution arising from the Lindblad approach to the one from the nonHermitian approach. Moreover, we propose a "hybrid" formalism that combines features of both of them, and, as such, it is expected to have a wider range of applicability. In order to illustrate the theory, we consider quantum two-level system (TLS) and study its time evolution while mimicking the coupling to a dissipative environment through non-Hermitian dynamics, Lindblad master equation, or their combination. The dynamics of the density matrix is solved analytically in a number of relevant cases, the behavior of primary observables (averages) is studied accordingly.

This paper is structured as follows. In section 2 we give a brief outline of the Lindblad and non-Hermitian approaches. In section 3 we combine them into a unified approach, named "hybrid" throughout the paper, and adopt a two-level system as a practical example. In sections 4, 5 and 6 we consider different limits of the hybrid dynamics of the two-level model, derive their analytical solutions and calculate the relevant observable properties. Some facts about two-level systems in quantum optics and relevant notations are reminded in the Appendix A. The definitions of the Fourier transforms used in the paper are given in the Appendix B. Conclusions are drawn in section 7

\section{OPEN QUANTUM SYSTEM DYNAMICS}

In this section we give a brief description of two popular density-operator based approaches which are used in a theory of open quantum systems. In both approaches, one distinguishes the coordinates of a subsystem from those of the environment so that the total Hamiltonian can be written as the sum $H_{\mathrm{T}}=H_{\mathrm{S}}+H_{\mathrm{B}}$ of the Hamiltonians of the relevant subsystem, $H_{\mathrm{S}}$, and of the environment (or bath), $H_{\mathrm{B}}$. Accordingly, the total Hilbert space becomes the product of two composing Hilbert spaces,

$$
\mathcal{H}_{\mathrm{T}}=\mathcal{H}_{\mathrm{S}} \otimes \mathcal{H}_{\mathrm{B}}
$$


where $\mathcal{H}_{\mathrm{S}}$ and $\mathcal{H}_{\mathrm{B}}$ are the Hilbert spaces of the relevant subsystem and bath, respectively. The density operator of the relevant subsystem is obtained by tracing out the degrees of freedom of the environment from the total density matrix:

$$
\hat{\rho}_{\mathrm{S}} \equiv \hat{\rho}=\operatorname{tr}_{B} \hat{\rho}_{\mathrm{T}},
$$

where $\operatorname{tr}_{\mathrm{B}}$ denotes the partial trace over the degrees of freedom of the environment $B$. Despite the common basis, the Lindblad and non-Hermitian approaches describe different effects of the environment onto the subsystem and produce different properties of the latter.

\subsection{Lindblad master equation}

This approach, while maintaining a Hamiltonian contribution to the dynamics of the subsystem, modifies the evolution equation of the subsystem density operator by adding dissipative terms (2). Upon using the Markov approximation and some auxiliary simplifications, one can show that the Lindblad master equation takes the form:

$$
\frac{d}{d t} \hat{\rho}(t)=-\frac{i}{\hbar}\left[\hat{H}_{+}, \hat{\rho}(t)\right]+\hat{\mathcal{D}}\left(\rho(t), A_{k}\right)
$$

where $\hat{H}_{+}=\hat{H}_{+}^{\dagger}$ is a Hamiltonian that commutes with that of the subsystem $\hat{H}_{\mathrm{S}}$. The dissipator $\hat{\mathcal{D}}\left(\rho, A_{k}\right)$ in Eq. (3) is a linear operator in $\hat{\rho}(t)$ and quadratic in the Lindblad operators $\hat{A}_{k}, k=1, \ldots, N^{2}-1$ and $N=\operatorname{dim}\left(\mathcal{H}_{S}\right)$. The dissipator must be traceless for the trace of the density operator to be conserved during evolution. The Lindblad operators $A_{k}$ describe directly the dissipative effects of the environment. The most general quantum dynamical semigroup form of the dissipator [9] can be written as

$$
\hat{\mathcal{D}}\left(\rho, A_{k}\right)=\sum_{k=1}^{N^{2}-1} \gamma_{k}\left(\hat{A}_{k} \hat{\rho} \hat{A}_{k}^{\dagger}-\frac{1}{2} \hat{A}_{k}^{\dagger} \hat{A}_{k} \hat{\rho}-\frac{1}{2} \hat{\rho} \hat{A}_{k}^{\dagger} \hat{A}_{k}\right)
$$

where $\gamma$ 's are non-negative quantities which can be expressed in terms of certain correlation functions of the environment and play the role of relaxation rates for different decay modes of the open subsystem.

Simple examples of the application of the Lindblad master equation are found when studying models of twolevel atoms interacting with the electromagnetic field in presence of a thermal reservoir of radiation modes [7 9$]$.

Using the notation of Appendix A we can write the master equation in the interaction picture as

$$
\frac{d}{d t} \hat{\rho}_{(I)}(t)=\frac{i \Omega}{2}\left[\hat{\sigma}_{+}+\hat{\sigma}_{-}, \hat{\rho}_{(I)}(t)\right]+\hat{\mathcal{D}}\left(\rho_{(I)}(t)\right),
$$

where the dissipator is given by

$$
\hat{\mathcal{D}}(\rho)=\gamma_{0}(N+1)\left[\hat{\sigma}_{-} \hat{\rho} \hat{\sigma}_{+}-\frac{1}{2}\left\{\hat{\sigma}_{+} \hat{\sigma}_{-}, \hat{\rho}\right\}\right]+\gamma_{0} N\left[\hat{\sigma}_{+} \hat{\rho} \hat{\sigma}_{-}-\frac{1}{2}\left\{\hat{\sigma}_{-} \hat{\sigma}_{+}, \hat{\rho}\right\}\right]
$$

where $\gamma_{0}$ is the spontaneous emission rate and $N=$ $N\left(\omega_{0}\right)$ denotes the Planck distribution at the transition frequency [9].

\subsection{Non-Hermitian approach}

Non-Hermitian dynamics has found various applications in the study of open quantum system [10 20]. Recently, it has been shown [19] that this approach is capable of describing the evolution of pure states into mixed ones - since the purity is not necessarily conserved if the dimension of the corresponding Hilbert space is larger than two. In turn, such a feature can be used in the quantitative modeling of the observer-related phenomena in quantum mechanics - such as the problem of measurement and phenomenon of decoherence. In the simplest formulation of this approach, it is assumed that the dissipative effects of the environment are somehow encoded in anti-Hermitian terms of the subsystem Hamiltonian which appear after averaging ("integrating out") the degrees of freedom of environment. Hence, in this approach one deals exclusively with the degrees of freedom of the subsystem, which is in turn described by a non-Hermitian Hamiltonian.

The non-Hermitian Hamiltonian operator can be always partitioned into Hermitian and anti-Hermitian parts

$$
\hat{H}=\hat{H}_{+}+\hat{H}_{-},
$$

where we denoted $\hat{H}_{ \pm}= \pm \hat{H}_{ \pm}^{\dagger}=\frac{1}{2}\left(\hat{H} \pm \hat{H}^{\dagger}\right)$. For further it is convenient to introduce also the self-adjoint operator $\hat{\Gamma} \equiv i \hat{H}_{-}$which will be referred as the decay rate operator throughout the paper. Starting from the Schrödinger equation, it is easy to show that the evolution equation for the density operator acquires an anticommutator term:

$$
\frac{d}{d t} \hat{\rho}(t)=-\frac{i}{\hbar}\left[\hat{H}_{+}, \hat{\rho}(t)\right]-\frac{i}{\hbar}\left\{\hat{H}_{-}, \hat{\rho}(t)\right\} .
$$

Equation (8) can also be written in matrix form [23], 
directly implementing, within a quantum framework, the original geometric ideas of Grmela [24] about dissipation.

While equations of the form (8) find some applications [14, 18], they also possess certain features (which also manifest themselves when working with the state vectors) that narrow their applicability range. For instance, the trace of the density operator determined by such equations is not preserved in general:

$$
\frac{d}{d t} \operatorname{tr}(\hat{\rho}(t))=\frac{2}{i \hbar} \operatorname{tr}\left(\hat{\rho}(t) \hat{H}_{-}\right) .
$$

This renders the usual probabilistic interpretation of quantum mechanics more problematic to achieve. Another issue arises if one studies the invariance of the evolution equation under the Hamiltonian "gauge" shift

$$
\hat{H} \rightarrow \hat{H}+c_{0} \hat{I},
$$

where $c_{0}$ is a c-number and $\hat{I}$ is an identity operator. As it happens in conventional quantum mechanics, one would like that such a shift should affect neither the observable averages nor the evolution equation. However, according to (8), this invariance gets broken if $c_{0}$ has an imaginary part.
In view of these circumstances, in our previous work [19] we proposed to consider the normalized density operator,

$$
\hat{\rho}^{\prime}=\hat{\rho} / \operatorname{tr}(\hat{\rho}),
$$

as a primary physical object of theory. Following this idea, the quantum average of an observable $\hat{O}=\hat{O}(0)$ is defined in terms of the normalized density operator in Eq. (11) as

$$
\langle O\rangle_{\mathrm{obs}} \equiv \operatorname{tr}\left(\hat{\rho}^{\prime} \hat{O}(0)\right)=\operatorname{tr}(\hat{\rho} \hat{O}(0)) / \operatorname{tr}(\hat{\rho}(t))
$$

This idea was also adopted in [20] where the evolution equation, which can be derived for the normalized density operator in our approach, was favored over the equations for the non-normalized operator and state vectors which were used previously (cf. [18], for instance). It turns out that the normalized density operator approach automatically solves the above-mentioned issues of normconservation and gauge invariance: using the evolution equation which follows from (8) and (11),

$$
\frac{d}{d t} \hat{\rho}^{\prime}(t)=-\frac{i}{\hbar}\left[\hat{H}_{+}, \hat{\rho}^{\prime}(t)\right]-\frac{i}{\hbar}\left\{\hat{H}_{-}, \hat{\rho}^{\prime}(t)\right\}+\frac{2 i}{\hbar} \operatorname{tr}\left(\hat{\rho}^{\prime}(t) \hat{H}_{-}\right) \hat{\rho}^{\prime}(t),
$$

one can easily check that the normalization property $\operatorname{tr}\left(\hat{\rho}^{\prime}\right)=1$ is conserved and that the "gauge" invariance under the transformation (10) is achieved for arbitrary $c_{0}$.

To conclude, the main advantage of the normalized density-operator approach in NHQM is that it handles in a unified way not only the pure states but also the mixed ones. Moreover, the emerging nonlinearity of the evolution equation (13) provides yet another example of a profound interplay between the physics of open quantum systems and nonlinear quantum mechanics: the environment is able to induce effective nonlinearities in quantum evolution equations 25,40$]$.

\section{3. "HYBRID" FORMALISM}

In this section we unify the Lindblad master equation with the non-Hermitian equation for the density matrix.
Such a hybrid equation is postulated replacing the usual Hamiltonian contribution to the evolution of the nonnormalized density matrix of the quantum subsystem in the Lindblad master equation with a more general nonHermitian one, taken from the $\mathrm{NH}$ equation (8). In such a way one obtains the following equation

$$
\frac{d}{d t} \hat{\rho}(t)=-\frac{i}{\hbar}\left[\hat{H}_{+}, \hat{\rho}(t)\right]-\frac{i}{\hbar}\left\{\hat{H}_{-}, \hat{\rho}(t)\right\}+\hat{\mathcal{D}}\left(\rho(t), A_{k}\right),
$$

where

$$
\hat{H}=\hat{H}_{+}+\hat{H}_{-}=\hat{H}_{+}-i \hat{\Gamma} \text {. }
$$

Upon substituting the normalized density operator (11) into equation (14), one obtains a a nonlinear evolution equation,

$$
\frac{d}{d t} \hat{\rho}^{\prime}(t)=-\frac{i}{\hbar}\left[\hat{H}_{+}, \hat{\rho}^{\prime}(t)\right]-\frac{i}{\hbar}\left\{\hat{H}_{-}, \hat{\rho}^{\prime}(t)\right\}+\hat{\mathcal{D}}\left(\rho^{\prime}(t), A_{k}\right)+\frac{2 i}{\hbar} \operatorname{tr}\left(\hat{\rho}^{\prime}(t) \hat{H}_{-}\right) \hat{\rho}^{\prime}(t) .
$$


Below it will be shown that this nonlinearity makes the models based on the hybrid equations substantially more interesting than those obtained from the Lindblad or nonHermitian equations alone.

In what follows, we study a two-level optical quantum system which is both an instructive example and a fruitful physical application. Using the notation and the definition of the system given in Appendix A. we assume that the evolution is governed by equations (14), (15) and (6). The model we study is defined by the following Hermitian Hamiltonian

$$
\hat{H}_{+}=\hat{H}_{0}+\hat{H}_{L},
$$

where

$$
\begin{aligned}
\hat{H}_{0} & =\frac{1}{2} \hbar \omega_{0} \hat{\sigma}_{3}, \\
\hat{H}_{L} & =\frac{1}{2} \hbar \Omega\left(\mathrm{e}^{-i \omega_{0} t} \hat{\sigma}_{+}+\mathrm{e}^{i \omega_{0} t} \hat{\sigma}_{-}\right) .
\end{aligned}
$$

The unperturbed Hamiltonian $\hat{H}_{0}$ can represent the two energy levels of a free dipole. In such a case, the perturbation $\hat{H}_{L}$ would describe the interaction between the dipole and a single-mode electromagnetic wave. More details and corresponding notations are provided in the Appendix A.

The anti-Hermitian Hamiltonian, which must be added to $\hat{H}_{+}$to give the total Hamiltonian $\hat{H}$ of the model, is

$$
\hat{H}_{-}=\hat{H}_{\Gamma}+\hat{H}_{D}+\hat{H}_{00},
$$

where

$$
\begin{aligned}
\hat{H}_{\Gamma} & =\frac{1}{2} i \hbar \Gamma \hat{\sigma}_{3}, \\
\hat{H}_{D} & =-\frac{1}{2} i \hbar \alpha\left(\mathrm{e}^{-i \omega_{0} t} \hat{\sigma}_{+}+\mathrm{e}^{i \omega_{0} t} \hat{\sigma}_{-}\right), \\
\hat{H}_{00} & =-\frac{1}{2} i \hbar \mathcal{T} \hat{I},
\end{aligned}
$$

where $\Gamma, \alpha$ and $\mathcal{T}$ are real-valued free parameters. The Hamiltonian $\hat{H}_{\Gamma} \propto \hat{\sigma}_{+} \hat{\sigma}_{-}$is motivated by the physics of photodetection and continuous measurements in presence of radiation modes, cf. Sec. 6.3.1 of [9]. The term $\hat{H}_{D}$ is the anti-Hermitian counterpart of $\hat{H}_{L}$, therefore, it is supposed to describe the dissipative processes accompanying the dipole interaction of the atom and electromagnetic field. The term $\hat{H}_{00}$ is a "gauge" term - as mentioned in the section 2.2, it does not affect observable values (as defined by (11) and (12)); however, it can be used for simplifying or regularizing intermediate expressions.

In terms of the above, the evolution equation of the model in the interaction picture reads

$$
\begin{aligned}
\frac{d}{d t} \hat{\rho}_{(I)} & =\frac{i \Omega}{2}\left[\hat{\sigma}_{+}+\hat{\sigma}_{-}, \hat{\rho}_{(I)}\right]-\frac{\alpha}{2}\left\{\hat{\sigma}_{+}+\hat{\sigma}_{-}, \hat{\rho}_{(I)}\right\} \\
& +\gamma_{0}(N+1)\left(\hat{\sigma}_{-} \hat{\rho}_{(I)} \hat{\sigma}_{+}-\frac{1}{2}\left\{\hat{\sigma}_{+} \hat{\sigma}_{-}, \hat{\rho}_{(I)}\right\}\right) \\
& +\gamma_{0} N\left(\hat{\sigma}_{+} \hat{\rho}_{(I)} \hat{\sigma}_{-}-\frac{1}{2}\left\{\hat{\sigma}_{-} \hat{\sigma}_{+}, \hat{\rho}_{(I)}\right\}\right) \\
& +\frac{\Gamma}{2}\left\{\hat{\sigma}_{3}, \hat{\rho}_{(I)}\right\}-\mathcal{T} \hat{\rho}_{(I)} .
\end{aligned}
$$

It is convenient to search for solutions of this equation in the form

$$
\begin{aligned}
\hat{\rho}_{(I)}(t) & =\frac{1}{2}\left[\hat{I} \operatorname{tr}\left(\hat{\rho}_{(I)}(t)\right)+\sum_{i=1}^{3} \hat{\sigma}_{i}\left\langle\sigma_{i}(t)\right\rangle_{(I)}\right] \\
= & \frac{1}{2}\left[\hat{I} \operatorname{tr}\left(\hat{\rho}_{(I)}(t)\right)+\hat{\sigma}_{3}\left\langle\sigma_{3}(t)\right\rangle_{(I)}\right] \\
& +\hat{\sigma}_{+}\left\langle\sigma_{-}(t)\right\rangle_{(I)}+\hat{\sigma}_{-}\left\langle\sigma_{+}(t)\right\rangle_{(I)}
\end{aligned}
$$

where

$$
\left\langle\sigma_{i}(t)\right\rangle_{(I)}=\operatorname{tr}\left(\hat{\sigma}_{i} \hat{\rho}_{(I)}(t)\right)
$$

with $i=1, \ldots, 3$, are auxiliary average values that are regarded as unknown functions of time, together with $\operatorname{tr}\left(\hat{\rho}_{(I)}(t)\right)=\operatorname{tr}(\hat{\rho}(t))$. The equations for the average values easily follow from equation (24)

$$
\begin{aligned}
& \frac{d}{d t}\langle\vec{\sigma}(t)\rangle_{(I)}=\mathbf{G}\langle\vec{\sigma}(t)\rangle_{(I)}+\vec{b} \operatorname{tr}\left(\hat{\rho}_{(I)}(t)\right), \\
& \frac{d}{d t} \operatorname{tr}\left(\hat{\rho}_{(I)}(t)\right)=-\alpha\left\langle\sigma_{1}(t)\right\rangle_{(I)}+\Gamma\left\langle\sigma_{3}(t)\right\rangle_{(I)} \\
&-\mathcal{T} \operatorname{tr}\left(\hat{\rho}_{(I)}(t)\right),
\end{aligned}
$$

where we have introduced the matrix

$$
\mathbf{G}=\left(\begin{array}{ccc}
-\frac{1}{2} \gamma-\mathcal{T} & 0 & 0 \\
0 & -\frac{1}{2} \gamma-\mathcal{T} & \Omega \\
0 & -\Omega & -\gamma-\mathcal{T}
\end{array}\right)
$$

and three-dimensional vector

$$
\vec{b}=\left(\begin{array}{c}
-\alpha \\
0 \\
\Gamma-\gamma_{0}
\end{array}\right)
$$

We have also adopted the vector notation $\vec{\sigma}=$ $\left(\sigma_{1}, \sigma_{2}, \sigma_{3}\right)$. It is very convenient to combine equations (27) and (28) into a single matrix equation for the four unknown functions, $\left\langle\sigma_{\mu}(t)\right\rangle_{(I)} \equiv$ $\left\{\langle\vec{\sigma}(t)\rangle_{(I)}, \operatorname{tr}\left(\hat{\rho}_{(I)}(t)\right)\right\}(\mu=1, \ldots, 4)$ :

$$
\frac{d}{d t}\left\langle\sigma_{\mu}(t)\right\rangle_{(I)}=\mathbf{M}\left\langle\sigma_{\mu}(t)\right\rangle_{(I)},
$$

where 


$$
\mathbf{M}=\left(\begin{array}{cccc}
-\frac{1}{2} \gamma-\mathcal{T} & 0 & 0 & -\alpha \\
0 & -\frac{1}{2} \gamma-\mathcal{T} & \Omega & 0 \\
0 & -\Omega & -\gamma-\mathcal{T} & \Gamma-\gamma_{0} \\
-\alpha & 0 & \Gamma & -\mathcal{T}
\end{array}\right), \hat{\sigma}_{\mu}=\left(\begin{array}{c}
\hat{\sigma}_{1} \\
\hat{\sigma}_{2} \\
\hat{\sigma}_{3} \\
\hat{I}
\end{array}\right)
$$

Equation (31) is in a matrix form that is useful in order to search for solutions. Nevertheless, it is instructive to re-write it in terms of normalized averages. Using equations (11) and (25), one can write the normalized density operator in a decomposed form

$$
\hat{\rho}_{(I)}^{\prime}(t)=\frac{1}{2}\left[\hat{I}+\sum_{i=1}^{3} \hat{\sigma}_{i}\left\langle\sigma_{i}^{\prime}(t)\right\rangle_{(I)}\right]=\frac{1}{2}\left[\hat{I}+\hat{\sigma}_{3}\left\langle\sigma_{3}^{\prime}(t)\right\rangle_{(I)}\right]+\hat{\sigma}_{+}\left\langle\sigma_{-}^{\prime}(t)\right\rangle_{(I)}+\hat{\sigma}_{-}\left\langle\sigma_{+}^{\prime}(t)\right\rangle_{(I)},
$$

where

$$
\left\langle\sigma_{i}^{\prime}(t)\right\rangle_{(I)}=\operatorname{tr}\left(\hat{\sigma}_{i} \hat{\rho}_{(I)}^{\prime}(t)\right)=\left\langle\sigma_{i}(t)\right\rangle_{(I)} / \operatorname{tr}(\hat{\rho}(t))
$$

$(i=1, \ldots, 3)$ are the observable average values in the interaction picture which satisfy the equation

$$
\frac{d}{d t}\left\langle\vec{\sigma}^{\prime}(t)\right\rangle_{(I)}=\mathbf{G}_{\mathrm{eff}}(t)\left\langle\vec{\sigma}^{\prime}(t)\right\rangle_{(I)}+\vec{b}
$$

where

$$
\mathbf{G}_{\mathrm{eff}}(t)=\mathbf{G}+\left(\mathcal{F}_{(I)}(t)+\mathcal{T}\right) \hat{I}=\left(\begin{array}{ccc}
\mathcal{F}_{(I)}(t)-\frac{1}{2} \gamma & 0 & 0 \\
0 & \mathcal{F}_{(I)}(t)-\frac{1}{2} \gamma & \Omega \\
0 & -\Omega & \mathcal{F}_{(I)}(t)-\gamma
\end{array}\right)
$$

and $\mathcal{F}_{(I)}(t) \equiv \alpha\left\langle\sigma_{1}^{\prime}(t)\right\rangle_{(I)}-\Gamma\left\langle\sigma_{3}^{\prime}(t)\right\rangle_{(I)}$. While this form of evolution equation is somewhat unsuitable for searching for analytical solutions (due to its non-linearity with respect to unknown functions $\left.\left\langle\sigma_{i}^{\prime}(t)\right\rangle_{(I)}\right)$, it allows us to demonstrate a feature mentioned in section 2.2 the contribution from the "gauge" term $\hat{H}_{00}$ disappears when one deals with observable values.

\section{GENERAL SOLUTION}

In this section, we search for solutions of Eq. (31) in the zero-temperature limit. This is equivalent to setting $\gamma=$ $\gamma_{0}$. Imposing the gauge condition $\mathcal{T}=0$ and rescaling the time variable $\tau=\Omega t$, we reduce (31) to the form:

$$
\frac{d}{d \tau}\left\langle\sigma_{\mu}(\tau)\right\rangle_{(I)}=\widetilde{\mathbf{M}}\left\langle\sigma_{\mu}(\tau)\right\rangle_{(I)}
$$

$(\mu=1, \ldots, 4)$ where

$$
\widetilde{\mathbf{M}}=\left(\begin{array}{cccc}
-2 \tilde{\gamma}_{0} & 0 & 0 & -\tilde{\alpha} \\
0 & -2 \tilde{\gamma}_{0} & 1 & 0 \\
0 & -1 & -4 \tilde{\gamma}_{0} & \tilde{\Gamma}-4 \tilde{\gamma}_{0} \\
-\tilde{\alpha} & 0 & \tilde{\Gamma} & 0
\end{array}\right),\left\langle\hat{\sigma}_{\mu}(\tau)\right\rangle=\left(\begin{array}{c}
\left\langle\hat{\sigma}_{1}(\tau)\right\rangle \\
\left\langle\hat{\sigma}_{2}(\tau)\right\rangle \\
\left\langle\hat{\sigma}_{3}(\tau)\right\rangle \\
\operatorname{tr} \rho(\tau)
\end{array}\right)
$$

In the definition of $\widetilde{\mathbf{M}}$, we have introduced the symbols $\tilde{\gamma}_{0}=\gamma_{0} / 4 \Omega, \tilde{\alpha}=\alpha / \Omega$ and $\tilde{\Gamma}=\Gamma / \Omega$. As in the previous sections, one should keep in mind that the nonnormalized values $\left\langle\sigma_{\mu}\right\rangle$ are auxiliary quantities that are used for computing the observables, equations (33) and

\section{(34).}

The solution of equation (37) can be formally written in a matrix exponential form:

$$
\left\langle\sigma_{\mu}(\tau)\right\rangle_{(I)}=e^{\widetilde{\mathbf{M}} \tau}\left\langle\sigma_{\mu}(0)\right\rangle_{(I)},
$$


where

$$
\left\langle\sigma_{\mu}(0)\right\rangle_{(I)}=\left\langle\sigma_{\mu}(0)\right\rangle=\left\langle\sigma_{\mu}^{\prime}(0)\right\rangle_{(I)}=\left\langle\sigma_{\mu}^{\prime}(0)\right\rangle
$$

are initial values. We used the property $\hat{\rho}(0)=\hat{\rho}^{\prime}(0)$, which is valid both in the Schrödinger and in the interaction picture. Naturally, one also finds that

$$
\left\langle\sigma_{4}(0)\right\rangle_{(I)}=\left\langle\sigma_{4}(0)\right\rangle=\left\langle\sigma_{4}^{\prime}(0)\right\rangle_{(I)}\left\langle\sigma_{4}^{\prime}(0)\right\rangle=\operatorname{tr} \hat{\rho}(0)=1,
$$

for all physical situations.

Furthermore, if the matrix $\widetilde{\mathbf{M}}$ is diagonalizable, the general solution (39) can be written in the more convenient form

$$
\begin{aligned}
& \left\langle\sigma_{\mu}(\tau)\right\rangle_{(I)}=\mathbf{S}\left\langle\sigma_{\mu}(0)\right\rangle_{(I)} \\
& \mathbf{S}=\mathbf{P}\left(\begin{array}{cccc}
e^{M_{(1)} \tau} & 0 & 0 & 0 \\
0 & e^{M_{(2)} \tau} & 0 & 0 \\
0 & 0 & e^{M_{(3)} \tau} & 0 \\
0 & 0 & 0 & e^{M_{(4)} \tau}
\end{array}\right) \mathbf{P}^{-1},(43
\end{aligned}
$$

where $M_{(\mu)}$ are eigenvalues of $\widetilde{\mathbf{M}}$, and $\mathbf{P}$ is the matrix whose columns are the eigenvectors of $\widetilde{\mathbf{M}}$. The four-byfour matrix $\widetilde{\mathbf{M}}$, with its four eigenvalues, arises from the hybrid master equation. Such an equation is defined in terms of both the dissipator and the total Hamiltonian $\hat{H}_{+}+\hat{H}_{-}$(which has just two eigenvalues for the model under study). Hence, the four eigenvalues of $\widetilde{\mathbf{M}}$ carry more physical information about the studied model system than the information carried by the eigenvalues of the total Hamiltonian alone.

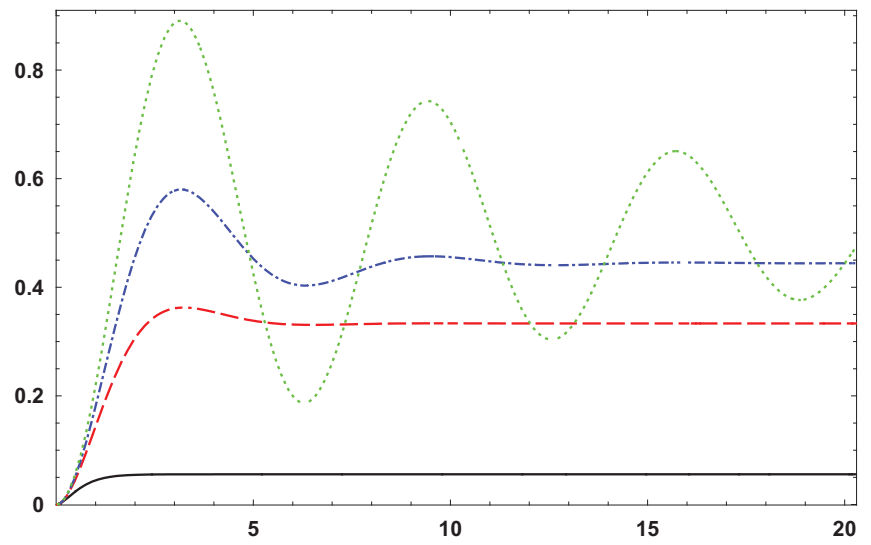

FIG. 1: The population of the upper level $p_{e}$ as a function of time $\tau=\Omega t$ for the parameters $\tilde{\alpha}=\tilde{\Gamma}=0$ and: $\tilde{\gamma}_{0}=1$ (solid curve), $\tilde{\gamma}_{0}=1 / 4$ (dashed curve), $\tilde{\gamma}_{0}=1 / 8$ (dash-dotted curve), and $\tilde{\gamma}_{0}=1 / 40$ (dotted curve).

\section{LIMIT CASES}

In this section we consider two special (limit) cases of the general solution found in section 4. In particular, we

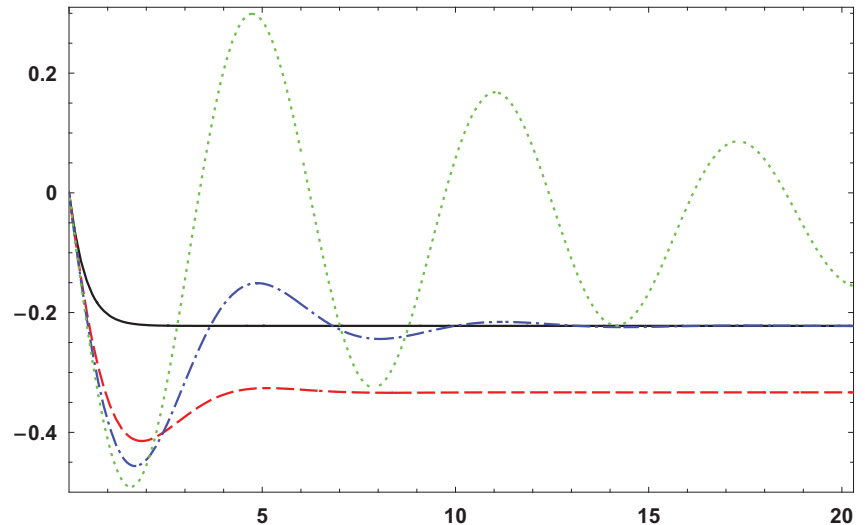

FIG. 2: The imaginary part of the coherence, $\operatorname{Im}\left(\left\langle\sigma_{+}\right\rangle_{\text {obs }}\right)$, as a function of time $\tau=\Omega t$ for the parameters $\tilde{\alpha}=\tilde{\Gamma}=0$ and: $\tilde{\gamma}_{0}=1$ (solid curve), $\tilde{\gamma}_{0}=1 / 4$ (dashed curve), $\tilde{\gamma}_{0}=1 / 8$ (dash-dotted curve), and $\tilde{\gamma}_{0}=1 / 40$ (dotted curve).

treat the case when dissipative effects are modeled either only by the Lindblad term (i.e., $\alpha=\Gamma=0$ ) and the case when only the anti-Hermitian term (i.e., $\gamma_{0}=0$ ) is present. This will allow us to obtain a clearer understanding of the capabilities of the hybrid formalism.

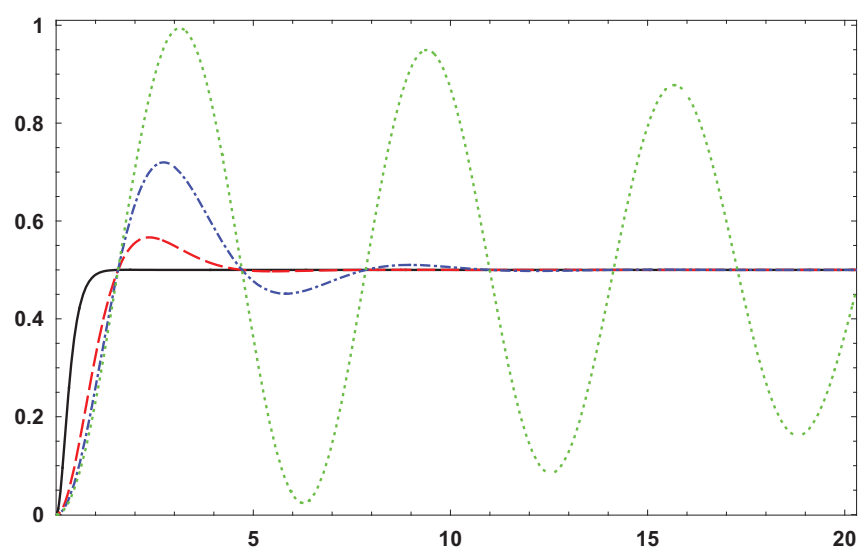

FIG. 3: The population of the upper level $p_{e}$ as a function of time $\tau=\Omega t$ for the parameters $\tilde{\gamma}_{0}=\tilde{\Gamma}=0$ and: $\tilde{\alpha}=4$ (solid curve), $\tilde{\alpha}=1$ (dashed curve), $\tilde{\alpha}=1 / 2$ (dash-dotted curve), and $\tilde{\alpha}=1 / 20$ (dotted curve).

\subsection{Lindblad-driven dissipation}

When $\alpha=\Gamma=0$, the matrix $\widetilde{\mathbf{M}}$ has the following four eigenvalues: 


$$
M_{1}^{(L)}=-2 \tilde{\gamma}_{0}, M_{2}^{(L)}=-3 \tilde{\gamma}_{0}-i \kappa, M_{3}^{(L)}=-3 \tilde{\gamma}_{0}+i \kappa, M_{4}^{(L)}=0
$$

where the quantity $\kappa=\sqrt{1-\tilde{\gamma}_{0}^{2}}$ can be imaginary or real-valued. Correspondingly, the solution from Sec. 4 can be written as

$$
\begin{aligned}
& \left\langle\sigma_{\mu}(\tau)\right\rangle_{(I)}=\mathbf{S}_{(L)}\left\langle\sigma_{\mu}(0)\right\rangle_{(I)}, \\
& \mathbf{S}_{(L)}=\left(\begin{array}{cccc}
e^{-2 \tilde{\gamma}_{0} \tau} & 0 & 0 & 0 \\
0 & f_{1}(\tau) e^{-3 \tilde{\gamma}_{0} \tau} & \frac{\sin (\kappa \tau)}{\kappa} e^{-3 \tilde{\gamma}_{0} \tau} & \frac{4 \tilde{\gamma}_{0}}{8 \tilde{\gamma}_{0}^{2}+1}\left(f_{3}(\tau) e^{-3 \tilde{\gamma}_{0} \tau}-1\right) \\
0 & -\frac{\sin (\kappa \tau)}{\kappa} e^{-3 \tilde{\gamma}_{0} \tau} & f_{-1}(\tau) e^{-3 \tilde{\gamma}_{0} \tau} & \frac{8 \tilde{\gamma}_{0}^{2}}{8 \tilde{\gamma}_{0}^{2}+1}\left(f_{-\nu^{2}}(\tau) e^{-3 \tilde{\gamma}_{0} \tau}-1\right) \\
0 & 0 & 0 & 1
\end{array}\right),
\end{aligned}
$$

where $f_{k}(\tau) \equiv \cos (\kappa \tau)+k \frac{\tilde{\gamma}_{0}}{\kappa} \sin (\kappa \tau)$ and $\nu^{2}=1+$ $1 /\left(2 \tilde{\gamma}_{0}^{2}\right)$. From the last row of this matrix it follows that $\left\langle\sigma_{4}(\tau)\right\rangle_{(I)}=\operatorname{tr}(\hat{\rho}(\tau))=1$. Therefore, the physical (normalized) averages coincide with the auxiliary ones: $\left\langle\sigma_{i}^{\prime}(t)\right\rangle=\left\langle\sigma_{i}(t)\right\rangle, i=1$...3. It is easy to check that this solution coincides with a textbook example - see, for instance, section 3.4.5.1 of [9] . The profiles of most important observables are given in the Figs. 1] and 2 .

The large-times asymptotic (steady-state) values of the spin averages can be found by taking an appropriate limit in equation (45). One obtains

$$
\left\langle\vec{\sigma}^{\prime}(+\infty)\right\rangle_{(I)}=\langle\vec{\sigma}(+\infty)\rangle_{(I)}=-\frac{4 \tilde{\gamma}_{0}}{8 \tilde{\gamma}_{0}^{2}+1}\left(\begin{array}{c}
0 \\
1 \\
2 \tilde{\gamma}_{0}
\end{array}\right) .
$$

These values are indeed stationary points of the system. Needless to say, they coincide with the textbook values, cf. section 3.4.5.1 of [9].

\subsection{Anti-Hermitian-driven dissipation}

When $\tilde{\gamma}_{0}=0$ then the matrix $\widetilde{\mathbf{M}}$ has the following four eigenvalues:

$$
\begin{aligned}
& \left(M_{1}^{(A)}, M_{2}^{(A)}, M_{3}^{(A)}, M_{4}^{(A)}\right)=\left(\lambda_{1}, \lambda_{2}, \lambda_{3}, \lambda_{4}\right)= \\
& \quad=\left(-\sqrt{\frac{R_{+}-R_{1}}{2}}, \sqrt{\frac{R_{+}-R_{1}}{2}},-\sqrt{\frac{R_{+}+R_{1}}{2}}, \sqrt{\frac{R_{+}+R_{1}}{2}},\right),
\end{aligned}
$$

where we have denoted $R_{1}=\sqrt{R_{+}^{2}+4 \tilde{\alpha}^{2}}=\sqrt{R_{-}^{2}+4 \tilde{\alpha}^{2} \tilde{\Gamma}^{2}}$ and $R_{ \pm}=\tilde{\alpha}^{2} \pm\left(\tilde{\Gamma}^{2}-1\right)$. One can see that $R_{1} \geqslant R_{ \pm}$so that the first two eigenvalues (47) are always imaginary-valued. Correspondingly, the solution from Sec. 4 can be written as

$$
\begin{aligned}
& \left\langle\sigma_{\mu}(\tau)\right\rangle_{(I)}=\mathbf{S}_{(A)}\left\langle\sigma_{\mu}(0)\right\rangle_{(I)}, \\
& \mathbf{S}_{(A)}=\frac{1}{R_{1}}\left(\begin{array}{cccc}
\frac{1}{2} C_{K_{-}, K_{+}}(\tau) & \tilde{\alpha} \tilde{\Gamma} S_{-\lambda_{2}^{-1}, \lambda_{4}^{-1}}(\tau) & \tilde{\alpha} \tilde{\Gamma} C_{1,-1}(\tau) & \tilde{\alpha} S_{\bar{\lambda}_{2},-\bar{\lambda}_{4}}(\tau) \\
\frac{\lambda_{2} \lambda_{4} \tilde{\Gamma}}{\tilde{\alpha}} S_{-\lambda_{4}, \lambda_{2}}(\tau) & C_{\lambda_{4} \bar{\lambda}_{4},-\lambda_{2} \bar{\lambda}_{2}}(\tau) & -\lambda_{2} \lambda_{4} S_{\bar{\lambda}_{4},-\bar{\lambda}_{2}}(\tau) & -\tilde{\Gamma} C_{1,-1}(\tau) \\
\tilde{\alpha} \tilde{\Gamma} C_{1,-1}(\tau) & \lambda_{2} \lambda_{4} S_{\bar{\lambda}_{4},-\bar{\lambda}_{2}}(\tau) & \frac{1}{2} C_{K_{+}, K_{-}}(\tau) & \tilde{\Gamma} S_{-\lambda_{2}, \lambda_{4}}(\tau) \\
-\frac{1}{2 \tilde{\alpha}} S_{\lambda_{2} K_{-}, \lambda_{4} K_{+}}(\tau) & \tilde{\Gamma} C_{1,-1}(\tau) & \tilde{\Gamma} S_{-\lambda_{2}, \lambda_{4}}(\tau) & C_{-\lambda_{2} \bar{\lambda}_{2}, \lambda_{4} \bar{\lambda}_{4}}(\tau)
\end{array}\right),
\end{aligned}
$$

where we have denoted

$$
\begin{aligned}
& C_{k_{1}, k_{2}}(\tau) \equiv k_{1} \cosh \left(\lambda_{2} \tau\right)+k_{2} \cosh \left(\lambda_{4} \tau\right)=k_{1} \cos \left(\left|\lambda_{2}\right| \tau\right)+k_{2} \cosh \left(\left|\lambda_{4}\right| \tau\right), \\
& S_{k_{1}, k_{2}}(\tau) \equiv k_{1} \sinh \left(\lambda_{2} \tau\right)+k_{2} \sinh \left(\lambda_{4} \tau\right)=i k_{1} \sin \left(\left|\lambda_{2}\right| \tau\right)+k_{2} \sinh \left(\left|\lambda_{4}\right| \tau\right)
\end{aligned}
$$


and $K_{ \pm}=R_{1} \pm R_{-}=\sqrt{R_{-}^{2}+4 \tilde{\alpha}^{2} \tilde{\Gamma}^{2}} \pm R_{-}$and $\bar{\lambda}_{k}=\lambda_{k}+1 / \lambda_{k}$. As before, the physical values are the normalized ones:

$$
\begin{aligned}
& \left\langle\vec{\sigma}^{\prime}(\tau)\right\rangle_{(I)}=\mathbf{S}_{(A)}^{\prime}\left\langle\sigma_{\mu}(0)\right\rangle_{(I)}, \\
& \mathbf{S}_{(A)}^{\prime}=\frac{1}{T_{(A)}}\left(\begin{array}{cccc}
\frac{1}{2} C_{K_{-}, K_{+}}(\tau) & \tilde{\alpha} \tilde{\Gamma} S_{-\lambda_{2}^{-1}, \lambda_{4}^{-1}}(\tau) & \tilde{\alpha} \tilde{\Gamma} C_{1,-1}(\tau) & \tilde{\alpha} S_{\bar{\lambda}_{2},-\bar{\lambda}_{4}}(\tau) \\
\frac{\lambda_{2} \lambda_{4} \tilde{\Gamma}}{\tilde{\alpha}} S_{-\lambda_{4}, \lambda_{2}}(\tau) & C_{\lambda_{4} \bar{\lambda}_{4},-\lambda_{2} \bar{\lambda}_{2}}(\tau) & -\lambda_{2} \lambda_{4} S_{\bar{\lambda}_{4},-\bar{\lambda}_{2}}(\tau) & -\tilde{\Gamma} C_{1,-1}(\tau) \\
\tilde{\alpha} \tilde{\Gamma} C_{1,-1}(\tau) & \lambda_{2} \lambda_{4} S_{\bar{\lambda}_{4},-\bar{\lambda}_{2}}(\tau) & \frac{1}{2} C_{K_{+}, K_{-}}(\tau) & \tilde{\Gamma} S_{-\lambda_{2}, \lambda_{4}}(\tau)
\end{array}\right),
\end{aligned}
$$

where $T_{(A)}$ is an internal product of the fourth row of $\mathbf{S}_{(A)}$ (omitting the overall factor) and the four-vector of initial values (40):

$$
T_{(A)}=-\frac{1}{2 \tilde{\alpha}} S_{\lambda_{2} K_{-}, \lambda_{4} K_{+}}(\tau)\left\langle\sigma_{1}(0)\right\rangle+\tilde{\Gamma} C_{1,-1}(\tau)\left\langle\sigma_{2}(0)\right\rangle+\tilde{\Gamma} S_{-\lambda_{2}, \lambda_{4}}(\tau)\left\langle\sigma_{3}(0)\right\rangle+C_{-\lambda_{2} \bar{\lambda}_{2}, \lambda_{4} \bar{\lambda}_{4}}(\tau) .
$$

Depending on whether the eigenvalue $\lambda_{4}$ vanishes or not, one can consider the following two cases.

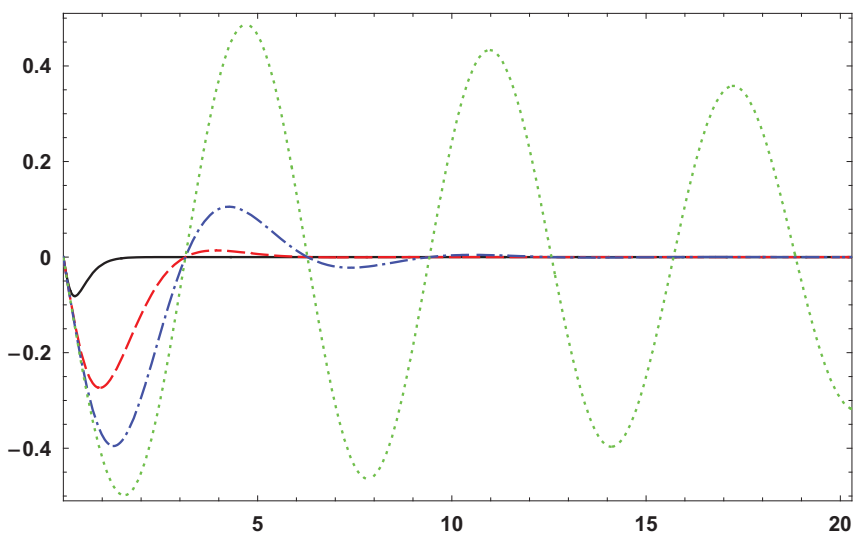

FIG. 4: The imaginary part of the coherence, $\operatorname{Im}\left(\left\langle\sigma_{+}\right\rangle_{\text {obs }}\right)$, as a function of time $\tau=\Omega t$ for the parameters $\tilde{\gamma}_{0}=\tilde{\Gamma}=0$ and: $\tilde{\alpha}=4$ (solid curve), $\tilde{\alpha}=1$ (dashed curve), $\tilde{\alpha}=1 / 2$ (dash-dotted curve), and $\tilde{\alpha}=1 / 20$ (dotted curve).

\subsubsection{Exponential damping}

This case takes place when the parameters of the model are such that $\lambda_{4} \neq 0$, or, alternatively, $\operatorname{sign}\left(R_{+}\right)+$ $\sqrt{1+\left(2 \tilde{\alpha} / R_{+}\right)^{2}} \neq 0$. Then the system exhibits the exponential damping which is qualitatively, but not necessarily quantitatively, similar to the Lindblad-driven dynamics.

Some generic profiles of most important observables are given in Figs. 3 and 4 (all curves), as well as in Figs. 5 and 6 (solid and dashed curves only). One can notice that Figs. 3 and 4 qualitatively resemble the Lindblad ones.

The large-times asymptotic values of the spin averages can be found by taking an appropriate limit in (49). At first one obtains

$$
\left\langle\vec{\sigma}^{\prime}(+\infty)\right\rangle_{(I)}=\frac{1}{\tilde{T}_{(A)}}\left(\begin{array}{cccc}
\frac{1}{2} \tilde{\alpha} K_{+} & \tilde{\alpha}^{2} \lambda_{4}^{-1} \tilde{\Gamma} & -\tilde{\alpha}^{2} \tilde{\Gamma} & \tilde{\alpha}^{2} \bar{\lambda}_{4} \\
\lambda_{2}^{2} \lambda_{4} \tilde{\Gamma} & -\tilde{\alpha} \lambda_{2} \bar{\lambda}_{2} & \tilde{\alpha} \lambda_{2} \bar{\lambda}_{2} \lambda_{4} & \tilde{\alpha} \tilde{\Gamma} \\
-\tilde{\alpha} \tilde{\Gamma} & -\tilde{\alpha} \lambda_{2} \bar{\lambda}_{2} \lambda_{4} & \frac{1}{2} \tilde{\alpha} K_{-} & \tilde{\alpha} \lambda_{4} \tilde{\Gamma}
\end{array}\right)\langle\vec{\sigma}(0)\rangle
$$

where we have denoted $\tilde{T}_{(A)}=-\frac{1}{2} \lambda_{4} K_{+}\left\langle\sigma_{1}(0)\right\rangle-$ $\tilde{\alpha} \tilde{\Gamma}\left\langle\sigma_{2}(0)\right\rangle+\tilde{\alpha} \lambda_{4} \tilde{\Gamma}\left\langle\sigma_{3}(0)\right\rangle+\tilde{\alpha} \lambda_{4} \bar{\lambda}_{4}$. Further simplifying this expression, we eventually obtain

$$
\left\langle\vec{\sigma}^{\prime}(+\infty)\right\rangle_{(I)}=\frac{1}{\lambda_{4} \bar{\lambda}_{4}}\left(\begin{array}{c}
-\tilde{\alpha} \bar{\lambda}_{4} \\
\tilde{\Gamma} \\
\lambda_{4} \tilde{\Gamma}
\end{array}\right),
$$

which means that the asymptotic (steady-state) averages do not depend on the initial values, as in the Lindblad case. One can see that the important difference from the Lindblad case is that $\left\langle\sigma_{x}^{\prime}\right\rangle_{(I)}$ does not vanish at large times.

Another distinctive features of the anti-Hermitiandriven dynamics can be found if one computes the Fourier transform of the observables, such as the population of an upper level $p_{e}$. The informative part of the Fourier 


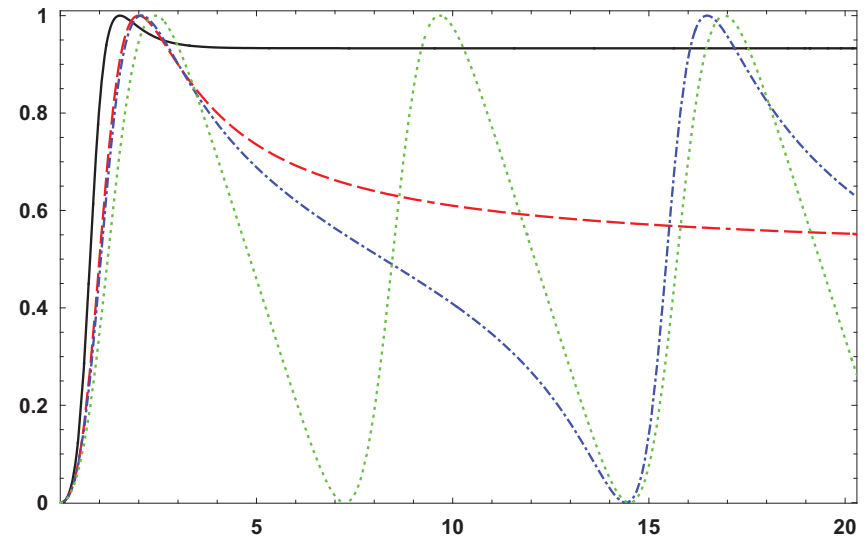

FIG. 5: The population of the upper level $p_{e}$ as a function of time $\tau=\Omega t$ for the parameters $\tilde{\alpha}=\tilde{\gamma}_{0}=0$ and: $\tilde{\Gamma}=2$ (solid curve), $\tilde{\Gamma}=1$ (dashed curve), $\tilde{\Gamma}=0.9$ (dash-dotted curve), and $\tilde{\Gamma}=1 / 2$ (dotted curve).

\subsubsection{Anharmonic oscillations}

In this case, the model parameters are such that $\lambda_{4}=0$, which is equivalent to the following two conditions: $\tilde{\alpha}=0$ and $\tilde{\Gamma}^{2}<1$. The solution exhibits purely oscillatory behavior:

$$
\left\langle\vec{\sigma}^{\prime}(\tau)\right\rangle_{(I)}=\frac{1}{T_{(A)}^{\Gamma}}\left(\begin{array}{c}
\omega_{\Gamma}^{2}\left\langle\sigma_{1}(0)\right\rangle \\
\tilde{\Gamma}-\tilde{\Gamma}^{2}\left\langle\sigma_{2}(0)\right\rangle-\left(\tilde{\Gamma}-\left\langle\sigma_{2}(0)\right\rangle\right) \cos \left(\omega_{\Gamma} \tau\right)+\omega_{\Gamma}\left\langle\sigma_{3}(0)\right\rangle \sin \left(\omega_{\Gamma} \tau\right) \\
\omega_{\Gamma}^{2}\left\langle\sigma_{3}(0)\right\rangle \cos \left(\omega_{\Gamma} \tau\right)+\left(\tilde{\Gamma}-\left\langle\sigma_{2}(0)\right\rangle\right) \sin \left(\omega_{\Gamma} \tau\right)
\end{array}\right)
$$

where the oscillation frequency $\omega_{\Gamma}=\sqrt{1-\tilde{\Gamma}^{2}}$, and $T_{(A)}^{\Gamma}=1-\tilde{\Gamma}\left\langle\sigma_{2}(0)\right\rangle-\tilde{\Gamma}\left(\tilde{\Gamma}-\left\langle\sigma_{2}(0)\right\rangle\right) \cos \left(\omega_{\Gamma} \tau\right)+$ $\omega_{\Gamma}\left\langle\sigma_{3}(0)\right\rangle \sin \left(\omega_{\Gamma} \tau\right)$.

Some profiles of most important observables are given in Figs. [5 and 6] (dotted and dash-dotted curves only). They exhibit interesting asymmetric oscillatory patterns which do not appear in the Lindblad case. Such patterns indicate a few important things. For instance, they show that the anti-Hermitian terms in the Hamiltonian can induce not only the standard decay effects (such as the asymptotic damping at large times) but also more sophisticated effects. Indeed, in this case the oscillations are not damped, the role of the anti-Hermitian parameter $\tilde{\Gamma}$ is that it introduces the asymmetry between the pumping and discharging of the two-level system. In terms of frequency it means that the pumping frequency is larger than the discharge one. It is similar to what happens in higher-than-two-level systems: first a system is pumped into the highest excited state, then it spontaneously cascades down to its ground state, passing the intermediate levels on its way. This is particularly clear to see when one takes a look at the Fourier transform of the population of the upper level $p_{e}$. The informative part of the
Fourier transform is, according to (B4),

$$
\left[p_{e}(n)\right]_{\mathrm{reg}} \propto \frac{1}{T_{o}} \int_{0}^{T_{o}} p_{e}(\tau) \mathrm{e}^{2 \pi i\left(n / T_{o}\right) \tau} d \tau
$$

where $T_{o}=2 \pi / \sqrt{1-\tilde{\Gamma}^{2}}$, and its specific functional form can be derived with the use of the equations (52), (A2) and (A14). The profile of the computed modulus of $\left[p_{e}(n)\right]_{\mathrm{reg}}$ is shown in the figure 9

To summarize, we have shown that the anti-Hermitian two-level models of this type can actually mimic the properties of quantum systems with more than two levels. In this picture the parameter $\tilde{\Gamma}$ turns out to be a qualitative measure of the number of the additional (effective) levels. For example, the figure 9 shows that the TLS with $\tilde{\Gamma}=1 / 2$ can be used to mimic the 4 -level or 5 -level system (if one neglects the "transitions" with the wavenumbers larger than four). The further decreasing of $\tilde{\Gamma}$ reduces the number of additional wave frequencies. 


\section{APPROXIMATE SOLUTION}

The general solution derived in Sec. 4 becomes extremely bulky when expressed in terms of radicals. Luckily, in some physical cases one could use certain approximations which drastically simplify final formulae. Indeed, in quantum-optical two-level systems the Rabi frequency usually takes large values, up to the megahertz scale, whereas the dissipative effects are small. It is thus natural to make the assumption

$$
\tilde{\gamma}_{0} \ll 1, \tilde{\alpha} \ll 1, \tilde{\Gamma} \ll 1,
$$

which corresponds to the strong driving limit, using the textbook terminology [9]. However, this condition is not enough since the perturbation theory has two different sectors - Lindblad-dominated (when the approximate series solution must converge to the solution from Sec. 5.1 when taking the limit $\alpha=\Gamma=0$ ) and anti-Hermitiandominated (when the series solution must converge to the solution from Sec. 5.2 in the limit $\gamma=0$ ). In the former case, which is of main interest here, one should supplement (54) with the assumption

$$
\bar{\alpha} \equiv \alpha / \gamma_{0} \ll 1, \bar{\Gamma} \equiv \Gamma / \gamma_{0} \ll 1
$$

then expand the exact solution (42) and the related observables in series with respect to these five small parameters, and keep the leading-order terms. By doing that we obtain that the matrix $\widetilde{\mathbf{M}}$ has the following four eigenvalues, in the leading-order approximation:

$$
\left(M_{1}^{(L D)}, M_{2}^{(L D)}, M_{3}^{(L D)}, M_{4}^{(L D)}\right) \approx\left(-2 \tilde{\gamma}_{0}-2 \tilde{\gamma}_{0} \chi_{2},-3 \tilde{\gamma}_{0}-i \chi_{1},-3 \tilde{\gamma}_{0}+i \chi_{1}, 2 \tilde{\gamma}_{0} \chi_{2}\right)
$$

where we denoted $\chi_{1}=1+(2 \bar{\Gamma}-1 / 2) \tilde{\gamma}_{0}^{2}$ and $\chi_{2}=\bar{\alpha}^{2} / 4$, both being positive-definite values. Correspondingly, the solution is given by

$$
\begin{aligned}
& \left\langle\sigma_{\mu}(\tau)\right\rangle_{(I)}=\mathbf{S}_{(L D)}\left\langle\sigma_{\mu}(0)\right\rangle_{(I)}, \\
& \mathbf{S}_{(L D)} \approx \mathbf{S}_{(L D)}^{(0)}+\mathbf{S}_{(L D)}^{(\alpha)}+\mathbf{S}_{(L D)}^{(\Gamma)}, \\
& \mathbf{S}_{(L D)}^{(0)}=\left(\begin{array}{cccc}
e^{-2 \tilde{\gamma}_{0}\left(1+\chi_{2}\right) \tau} & 0 & 0 & 0 \\
0 & h_{+}(\tau) e^{-3 \tilde{\gamma}_{0} \tau} & \sin \left(\chi_{1} \tau\right) e^{-3 \tilde{\gamma}_{0} \tau} & 4 \tilde{\gamma}_{0} h_{2}(\tau) \\
0 & -\sin \left(\chi_{1} \tau\right) e^{-3 \tilde{\gamma}_{0} \tau} & h_{-}(\tau) e^{-3 \tilde{\gamma}_{0} \tau} & -4 \tilde{\gamma}_{0} \sin \left(\chi_{1} \tau\right) e^{-3 \tilde{\gamma}_{0} \tau} \\
0 & 0 & 0 & e^{2 \tilde{\gamma}_{0} \chi_{2} \tau}
\end{array}\right) \\
& \mathbf{S}_{(L D)}^{(\alpha)}=\bar{\alpha}\left(\begin{array}{cccc}
0 & 0 & 0 & h_{1}(\tau) \\
-4 \tilde{\gamma}_{0} h_{1}(\tau) & 0 & 0 & 0 \\
-4 \tilde{\gamma}_{0}^{2} h_{2}(\tau) & 0 & 0 & 0 \\
h_{1}(\tau) & 0 & 0 & 0
\end{array}\right) \\
& \mathbf{S}_{(L D)}^{(\Gamma)}=\tilde{\Gamma}\left(\begin{array}{cccc}
0 & -\bar{\alpha} h_{1}(\tau) & \tilde{\alpha} h_{2}(\tau) & 0 \\
\bar{\alpha} h_{1}(\tau) & 0 & 0 & -h_{2}(\tau) \\
\tilde{\alpha} h_{2}(\tau) & 0 & 0 & \sin \left(\chi_{1} \tau\right) e^{-3 \tilde{\gamma}_{0} \tau} \\
0 & h_{2}(\tau) & \sin \left(\chi_{1} \tau\right) e^{-3 \tilde{\gamma}_{0} \tau} & 0
\end{array}\right)
\end{aligned}
$$

where $h_{1}(\tau)=\frac{1}{2}\left(e^{-2 \tilde{\gamma}_{0}\left(1+\chi_{2}\right) \tau}-e^{2 \tilde{\gamma}_{0} \chi_{2} \tau}\right), h_{2}(\tau)=\cos \left(\chi_{1} \tau\right) e^{-3 \tilde{\gamma}_{0} \tau}-e^{2 \tilde{\gamma}_{0} \chi_{2} \tau}$, and $h_{ \pm}(\tau)=\cos \left(\chi_{1} \tau\right) \pm \tilde{\gamma}_{0} \sin \left(\chi_{1} \tau\right)$. As in Sec. 5.2, the physical values are the normalized ones:

$$
\left\langle\vec{\sigma}^{\prime}(\tau)\right\rangle_{(I)}=\frac{1}{T_{(L D)}} \mathbf{S}_{(L D)}^{\prime}\left\langle\sigma_{\mu}(0)\right\rangle_{(I)}
$$

where $\mathbf{S}_{(L D)}^{\prime}$ is the matrix $\mathbf{S}_{(L D)}$ without the bottom row, $T_{(L D)}$ is a product of the bottom row of $\mathbf{S}_{(L D)}$ and the four-vector of initial values (40):

$$
T_{(L D)}=\bar{\alpha} h_{1}(\tau)\left\langle\sigma_{1}(0)\right\rangle+\tilde{\Gamma} h_{2}(\tau)\left\langle\sigma_{2}(0)\right\rangle+\tilde{\Gamma} \sin \left(\chi_{1} \tau\right) e^{-3 \tilde{\gamma}_{0} \tau}\left\langle\sigma_{3}(0)\right\rangle+e^{2 \tilde{\gamma}_{0} \chi_{2} \tau} .
$$

The large-times asymptotic values of the spin averages can be found by taking an appropriate limit in (59).
Hence, we obtain in the leading-order approximation

$$
\left\langle\vec{\sigma}^{\prime}(+\infty)\right\rangle_{(I)}=\left(\begin{array}{c}
-\frac{1}{2} \bar{\alpha}-2 \tilde{\alpha} \tilde{\Gamma} \\
\Gamma-4 \tilde{\gamma}_{0} \\
2 \tilde{\gamma}_{0}\left(\tilde{\Gamma}-4 \tilde{\gamma}_{0}\right)
\end{array}\right)
$$




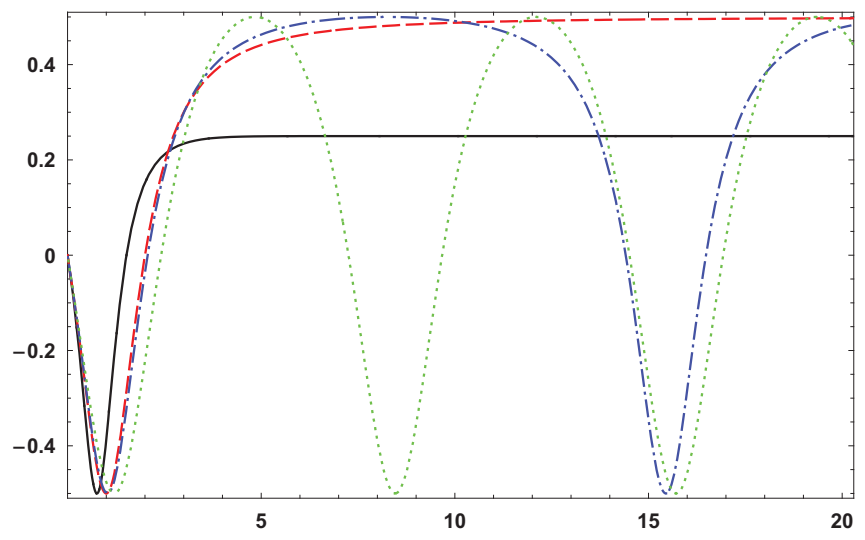

FIG. 6: The imaginary part of the coherence, $\operatorname{Im}\left(\left\langle\sigma_{+}\right\rangle_{\mathrm{obs}}\right)$, as a function of time $\tau=\Omega t$ for the parameters $\tilde{\alpha}=\tilde{\gamma}_{0}=0$ and: $\tilde{\Gamma}=2$ (solid curve), $\tilde{\Gamma}=1$ (dashed curve), $\tilde{\Gamma}=0.9$ (dash-dotted curve), and $\tilde{\Gamma}=1 / 2$ (dotted curve).

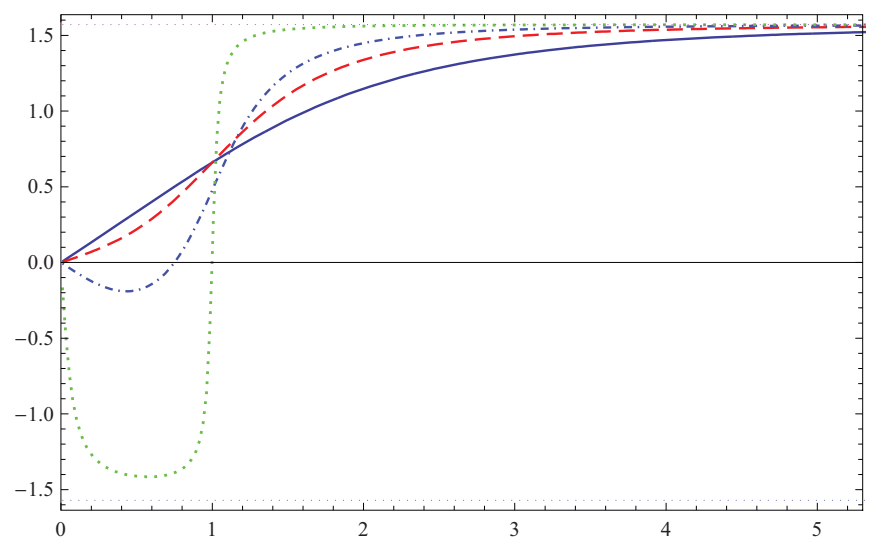

FIG. 7: The phase of the Fourier transform $p_{e}(\omega)$ versus the frequency $\tilde{\omega}=\omega / \Omega$, for the parameters $\tilde{\alpha}=\tilde{\Gamma}=0$ and: $\tilde{\gamma}_{0}=1 / 2$ (solid curve), $\tilde{\gamma}_{0}=1 / 4$ (dashed curve), $\tilde{\gamma}_{0}=1 / 8$ (dash-dotted curve), and $\tilde{\gamma}_{0}=1 / 100$ (dotted curve). Two horizontal thin dotted lines mark the values $\pm \pi / 2$.

\section{CONCLUSION}

In this paper we have compared two approaches to describing the effects of a general dissipative environment. Namely, we considered both the approach based on the Lindblad master equation and the formalism based on introducing anti-Hermitian terms into the Hamiltonian. In section 3 we have proposed a "hybrid" formalism that unifies the Lindblad and non-Hermitian approaches. This allowed us not only to reveal the distinctive features of the approaches but also to expand the range of dissipative phenomena that can be accounted for.

Using a two-level single-atom model as a practical application, we have obtained solutions of the hybrid equa-

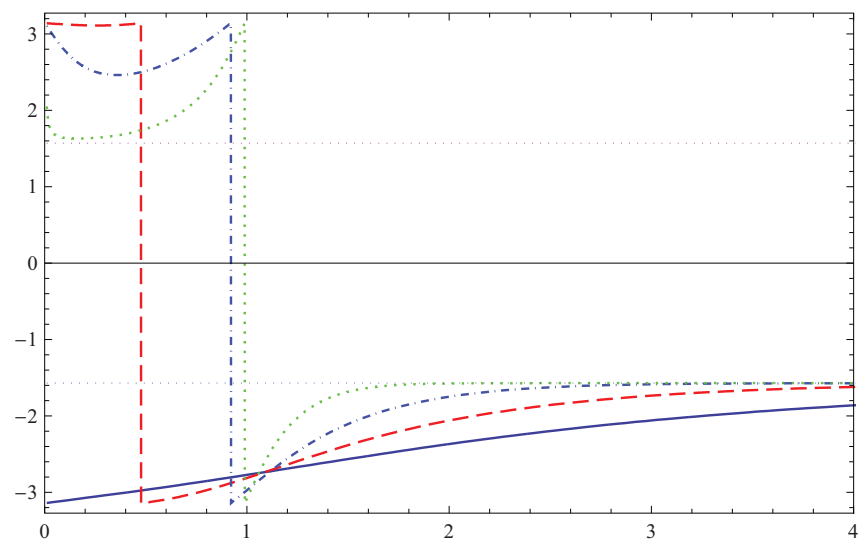

FIG. 8: The phase of the Fourier transform $p_{e}(\omega)$ (51) versus the frequency $\tilde{\omega}=\omega / \Omega$, for the parameters $\tilde{\gamma}_{0}=\tilde{\Gamma}=0$ and: $\tilde{\alpha}=2$ (solid curve), $\tilde{\alpha}=1$ (dashed curve), $\tilde{\alpha}=1 / 2$ (dashdotted curve), and $\tilde{\alpha}=1 / 5$ (dotted curve). Two horizontal thin dotted lines mark the values $\pm \pi / 2$.

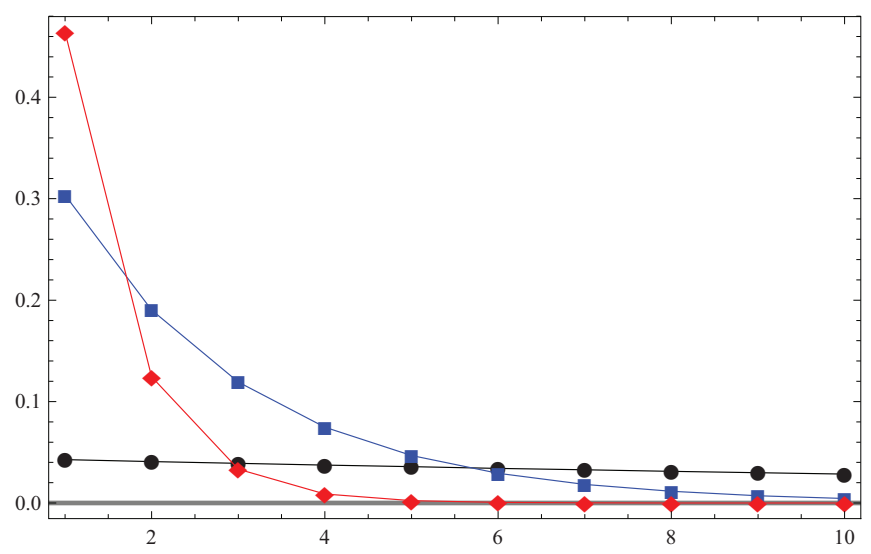

FIG. 9: The modulus of the Fourier transform $p_{e}(n)$ (53) versus the wavenumber $n$, for the parameters $\tilde{\alpha}=\tilde{\gamma}_{0}=0$ and: $\tilde{\Gamma}=0.999$ (dots), $\tilde{\Gamma}=0.9$ (squares), and $\tilde{\Gamma}=1 / 2$ (diamonds).

tion for the normalized density matrix operator. In sections 4, 5 and 6. we have also considered special (limit) cases and physically admissible approximations. Using the analytical solutions of all these cases, we have calculated those properties of the model that can be compared with experiments in order to assess whether a specific feature is either non-Hermitian driven or Lindblad driven.

Remarkably, we have found that the anti-Hermitian terms in the Hamiltonian can describe not only the mere dissipative damping but also undamped anharmonic oscillatory phenomena. Such results are reported in detail in section 5.2 where we also showed that this kind of anharmonicity can be used to mimic the cascaded quantum systems with more than two levels. In future it would be interesting to apply the hybrid formalism to those multi-level lasers or spin systems that can be modeled 
in the leading order of approximation by means of only two states. In particular, we have in mind those systems where one of the two energy levels is actually a band or a bundle of a few closely situated levels.

\section{Acknowledgments}

This article is based on the talks given at the conferences "12th International Workshop on PseudoHermitian Hamiltonians in Quantum Physics" (02-06 July, 2013, Koç University, Istanbul, Turkey) and "Quantum Information Processing, Communication and Control 2" (25-29 November, 2013, KwaZulu-Natal, South Africa). This work was supported by the National Research Foundation of South Africa.

\section{Appendix A: Two-level systems in quantum optics}

The two-mode open quantum system is a basic yet very instructive example of an open quantum (sub)system. In quantum optics its most obvious manifestation is the twolevel atom interacting with the external electromagnetic field (such as the laser field) and dissipative environment (heat bath, noise, etc). However, two-level models can also serve as a decent first-order approximation for those physical phenomena whose dynamics is effectively confined to a two-dimensional subspace, one example to be the systems for which one can neglect the influence of excited levels above the first excited one. Here we outline the basic notions used in a theory of two-level quantum optical systems.

For a general two-level quantum system the Hilbert space has the dimensionality two, and it is spanned by just two states, a ground state $|g\rangle$ and an excited state $|e\rangle$. An arbitrary quantum state of such system can be written in the basis of the Pauli and unit matrices which form a complete set. In quantum optics one is often interested in such averaged values as the population difference

$$
\left\langle\sigma_{3}\right\rangle_{\mathrm{obs}} \equiv \operatorname{tr}\left(\hat{\rho} \hat{\sigma}_{3}\right) / \operatorname{tr}(\hat{\rho})=\frac{\rho_{11}-\rho_{22}}{\rho_{11}+\rho_{22}},
$$

the population of the excited-state (upper) level

$$
p_{e}=\frac{\rho_{11}}{\rho_{11}+\rho_{22}}=\frac{1}{2}\left(1+\left\langle\sigma_{3}\right\rangle_{\mathrm{obs}}\right)
$$

the population of the ground state level

$$
p_{g}=1-p_{e}=\frac{1}{2}\left(1-\left\langle\sigma_{3}\right\rangle_{\mathrm{obs}}\right),
$$

and the coherence

$$
\left\langle\sigma_{+}\right\rangle_{\text {obs }} \equiv \operatorname{tr}\left(\hat{\rho} \hat{\sigma}_{+}\right) / \operatorname{tr}(\hat{\rho}),
$$

where $\rho_{i j}$ are the $i j$ th components of the density matrix. One can check that during the evolution the spin averages obey the following identity

$$
\left\langle\sigma_{1}\right\rangle_{\mathrm{obs}}^{2}+\left\langle\sigma_{2}\right\rangle_{\mathrm{obs}}^{2}+\left\langle\sigma_{3}\right\rangle_{\mathrm{obs}}^{2}=1-4 \operatorname{det}(\hat{\rho} / \operatorname{tr} \hat{\rho}) \leqslant 1
$$

which means that for pure states the averages lie on the Bloch sphere $\left\langle\sigma_{1}\right\rangle_{\text {obs }}^{2}+\left\langle\sigma_{2}\right\rangle_{\text {obs }}^{2}+\left\langle\sigma_{3}\right\rangle_{\text {obs }}^{2}=1$.

The unperturbed Hamiltonian of a quantum-optical two-level system is usually a linear combination of the operators $|g\rangle\langle g|$ and $|e\rangle\langle e|$. Up to an additive constant it equals to

$$
\hat{H}_{0}=\frac{1}{2} \hbar \omega_{0} \hat{\sigma}_{3}
$$

where $\omega_{0}$ is the transition frequency. If the system is put into contact with a monochromatic electromagnetic wave of frequency $\omega_{0}$ then in the leading order we can restrict ourselves with the dipole interaction. In the rotationwave approximation (RWA) the corresponding Hamiltonian can be reduced to the form

$$
\hat{H}_{L}=\frac{1}{2} \hbar \Omega\left(\mathrm{e}^{-i \omega_{0} t} \hat{\sigma}_{+}+\mathrm{e}^{i \omega_{0} t} \hat{\sigma}_{-}\right),
$$

where $\Omega$ is the Rabi frequency which measures the strength of the interaction of the system's dipole moment with the electromagnetic field, and $\hat{\sigma}_{ \pm}=\frac{1}{2}\left(\hat{\sigma}_{1} \pm i \hat{\sigma}_{2}\right)$.

For the purposes of simplifying the evolution equations it is often very convenient to perform a transition from the Schrödinger picture to the interaction one. One starts with the unitary transformation of the density operator

$$
\hat{\rho}_{(I)}=\mathrm{e}^{i \hat{H}_{0} t / \hbar} \hat{\rho} \mathrm{e}^{-i \hat{H}_{0} t / \hbar}
$$

where $\hat{H}_{0}$ is chosen as in (A6). This implies the transition formulae

$$
\begin{aligned}
& \operatorname{tr} \hat{\rho}_{(I)}=\operatorname{tr} \hat{\rho}, \\
& \operatorname{tr}\left(\hat{\rho}_{(I)} \hat{O}^{(I)}\right)=\operatorname{tr}(\hat{\rho} \hat{O}), \\
& \hat{O}^{(I)}=\mathrm{e}^{i \hat{H}_{0} t / \hbar} \hat{O} \mathrm{e}^{-i \hat{H}_{0} t / \hbar},
\end{aligned}
$$

where $\hat{O}$ refers to an observable's operator, the label $(I)$ indicates the interaction picture with respect to $\hat{H}_{0}$, and absence of the label denotes the Schrödinger picture presentation. Using the expressions above and Pauli matrices' properties, we can write down the following transformation chart between the Schrödinger and interaction pictures to be used in the evolution equations of the type (3) or (8):

$$
\begin{aligned}
& \hat{\rho} \mapsto \hat{\rho}_{(I)}, \quad\left[\hat{H}_{0}, \hat{\rho}\right] \mapsto 0, \\
& \hat{H}_{L} \mapsto \frac{1}{2} \hbar \Omega\left(\hat{\sigma}_{+}+\hat{\sigma}_{-}\right)=\frac{1}{2} \hbar \Omega \hat{\sigma}_{1}, \\
& \vec{\sigma} \mapsto\left(\begin{array}{ccc}
\cos \left(\omega_{0} t\right) & \sin \left(\omega_{0} t\right) & 0 \\
-\sin \left(\omega_{0} t\right) & \cos \left(\omega_{0} t\right) & 0 \\
0 & 0 & 1
\end{array}\right) \vec{\sigma}, \\
& \hat{\sigma}_{ \pm} \mapsto \mathrm{e}^{ \pm i \omega_{0} t} \hat{\sigma}_{ \pm},
\end{aligned}
$$

where by $\vec{\sigma}$ and $\vec{\sigma}^{(I)}$ we denote a set of the three Pauli operators in the Schrödinger and interaction picture, respectively. 


\section{Appendix B: Fourier transform in open quantum} systems

Let us consider the following setup: some observable, $F(t)$, evolves according to quantum evolution equations. Suppose that in absence of background effects its value is trivial: $F(t<0)=f_{c}=$ const. Then at a certain moment of time, say $t=0$, one "switches on" the background effects, such that the total function becomes the following:

$$
F(t)=f_{c} \theta(-t)+f(t) \theta(t)
$$

where $\theta$ is the Heaviside step function and $f(t)=F(t \geqslant$ $0)$. For practical purposes we will be interested in the following two scenarios:

(a) function $f(t)$ tends to a constant value $f_{\infty}$ at $t \rightarrow$ $+\infty$.

In this case the Fourier transform of the global function can be written as

$$
F(\omega)=\frac{1}{\sqrt{2 \pi}}\left(f_{c}+f_{\infty}\right) \delta(\omega)+[F(\omega)]_{\mathrm{reg}}
$$

where $[F(\omega)]_{\text {reg }}$ is the regular part of the Fourier transform:

$$
[F(\omega)]_{\mathrm{reg}}=\frac{1}{\sqrt{2 \pi}} \int_{0}^{\infty}\left(f(t)-f_{\infty}\right) \mathrm{e}^{i \omega t} d t
$$

which is going to be the most informative for our purposes.

(b) function $f(t)$ oscillates with a period $T$.

In this case the regular part of the Fourier transform of the global function can be computed as

$$
[F(n)]_{\mathrm{reg}}=\frac{1}{T} \int_{0}^{T} f(t) \mathrm{e}^{2 \pi i(n / T) t} d t
$$

where $n$ is an integer positive number.
[1] S. Attal and A. Joye, in: Open Quantum Systems I: The Hamiltonian Approach, eds. C.-A. Pillet (Springer, Berlin, 2006).

[2] A. Sergi, I. Sinayskiy, and F. Petruccione, Phys. Rev. A 80, 012108 (2009).

[3] V. Gorini, A. Kossakowski and E. C. G. Sudarshan, J. Math. Phys. 17, 821-825 (1976).

[4] G. Lindblad, Commun. Math. Phys. 48, 119-130 (1976).

[5] R. Bausch, Z. Phys. 193, 246-265 (1966).

[6] F. Haake, Springer Tracts Mod. Phys. 66, 98-168 (1973).

[7] H. J. Carmichael, An Open Systems Approach to Quantum Optics, Lecture Notes in Physics (Springer-Verlag, Berlin, 1993).

[8] C. W. Gardiner and P. Zoller, Quantum Noise (SpringerVerlag, Berlin, 2000).

[9] H.-P. Breuer and F. Petruccione, The Theory of Open Quantum Systems (Oxford University Press, 2002).

[10] H. Feshbach, Ann. Phys. 5, 357-390 (1958); ibid. 19, $287-$ $313(1962)$

[11] J. Wong, J. Math. Phys. 8, 2039-2042 (1967).

[12] F. H. M. Faisal and J. V. Moloney, J. Phys. B: At. Mol. Opt. Phys. 14, 3603-3620 (1981).

[13] G. Dattoli, A. Torre, and R. Mignani, Phys. Rev. A 42, 1467-1475 (1990).

[14] G. C. Hegerfeldt, Phys. Rev. A 47, 449-455 (1993).

[15] S. Baskoutas et al., J. Phys. A: Math. Gen. 26, L819L824 (1993); P. Angelopoulou et al., Int. J. Mod. Phys. B 9, 2083-2104 (1995).

[16] I. Rotter, arXiv:0711.2926 J. Phys. A 42, 153001 (2009).

[17] H. B. Geyer, F. G. Scholtz and K. G. Zloshchastiev, in: Proceedings of $12^{\text {th }}$ International Conference on Mathematical Methods in Electromagnetic Theory (Odessa, 2008) 250-252.

[18] E.-M. Graefe and R. Schubert, Phys. Rev. A 83, 060101
(2011); J. Phys. A 45, 244033 (2012).

[19] A. Sergi and K. G. Zloshchastiev, Int. J. Mod. Phys. B 27, 1350163 (2013) arXiv:1207.4877.

[20] D. C. Brody and E.-M. Graefe, Phys. Rev. Lett. 109, 230405 (2012) arXiv:1208.5297.

[21] E.-M. Graefe, M. Höning, and H. J. Korsch, J. Phys. A 43, 075306 (2010).

[22] A. Thilagam, J. Chem. Phys. 136, 065104 (2011).

[23] A. Sergi, Comm. Theor. Phys. 56, 96-98 (2011).

[24] M. Grmela, Phys. Lett. A 102, 355-358 (1984).

[25] N. Gisin, J. Phys. A 14, 2259-2267 (1981).

[26] N. Gisin, Physica A 111, 364-370 (1982).

[27] N. Gisin, J. Math. Phys. 24, 1779-1782 (1983).

[28] H. J. Korsch and H. Steffen, J. Phys. A 20, 3787-3803 (1987).

[29] M. D. Kostin, J. Chem. Phys. 57, 3589-3591 (1972).

[30] M. D. Kostin, J. Stat. Phys. 12, 145-151 (1975).

[31] I. Bialynicki-Birula and J. Mycielski, Annals Phys. 100, 62-93 (1976).

[32] K. Yasue, Annals Phys. 114, 479-496 (1978).

[33] N. A. Lemos, Phys. Lett. A 78, 239-241 (1980).

[34] J. D. Brasher, Int. J. Theor. Phys. 30, 979-984 (1991).

[35] D. Schuch, Phys. Rev. A 55, 935-940 (1997).

[36] M. P. Davidson, Nuov. Cim. B 116, 1291-1294 (2001).

[37] J. L. Lopez, Phys. Rev. E. 69, 026110 (2004).

[38] K. G. Zloshchastiev, Grav. Cosmol. 16, 288-297 (2010).

[39] A. V. Avdeenkov and K. G. Zloshchastiev, J. Phys. B: At. Mol. Opt. Phys. 44, 195303 (2011) arXiv:1108.0847.

[40] K. G. Zloshchastiev, Eur. Phys. J. B 85, 273 (2012) arXiv:1204.4652. 